ESJ Humanities

\title{
Medición de Emociones en Los Jóvenes Hacia Emprendimientos de Bebidas de Guayusa: Implicaciones de Gestión para la Comercialización de Productos
}

\author{
Dayana Jamile Romero-Buele \\ Ingeniera en Administración de Empresas de la Universidad Técnica de \\ Machala (UTMACH), Integrante del grupo de investigación OroResearch de \\ la UTMACH, Dirección: Machala, El Oro, Ecuador
}

Diana Maribel Domínguez-Maldonado

Ingeniera en Administración de Empresas de la Universidad Técnica de Machala (UTMACH), Integrante del grupo de investigación OroResearch de la UTMACH, Dirección: Machala, El Oro, Ecuador

\section{Raquel Miroslava Tinoco-Egas}

Doctora en Análisis Económico y Estrategia Empresarial por la Universidade da Coruña, España, Master of Science in International Business

Development, Université de Neuachâtel, Suiza, Ingeniera en Gestión Empresarial Internacional, Profesora Titular de la Facultad de Ciencias

Empresariales de la Universidad Técnica de Machala (UTMACH), Coordinadora del grupo de investigación OroResearch de la UTMACH, Dirección: Machala, El Oro, Ecuador

\section{Jonathan Neptalí Herrera-Peña}

Doctor en Análisis Económico y Estrategia Empresarial por la Universidade da Coruña, España, Magíster en Administración de Empresas por la Universidad Técnica de Machala (UTMACH), Diplomado Superior en Docencia Universitaria por la Universidad Técnica de Machala (UTMACH), Docente Titular de la Facultad de Ciencias Empresariales de la Universidad Técnica de Machala (UTMACH), Integrante del grupo de investigación OroResearch de la UTMACH, Dirección: Machala, El Oro, Ecuador

Doi:10.19044/esj.2021.v17n39p42

Submitted: 25 November 2020

Accepted: 01 November 2021

Published: 30 November 2021
Copyright 2021 Author(s)

Under Creative Commons BY-NC-ND

4.0 OPEN ACCESS

Cite As:

Romero-Buele D.J., Domínguez-Maldonado D.M., Tinoco-Egas R.M. \& Herrera-Peña J.N. (2021). Medición de Emociones en Los Jóvenes Hacia Emprendimientos de Bebidas de Guayusa: Implicaciones de Gestión para la Comercialización de Productos. European Scientific Journal, ESJ, 17 (39), 42. https://doi.org/10.19044/esj.2021.v17n39p42 


\section{Resumen}

La métrica de las emociones a partir de dispositivos de Neuromarketing valida las respuestas de comportamiento del consumidor desde el cerebro ante los estímulos sensoriales, en este caso, el empaque a través de la vista, y el sabor mediante el gusto de las bebidas energéticas naturales, como método analítico de la neurociencia para la aprobación de nuevos productos en el mercado. Esta investigación exploratorio-experimental tiene como objetivo medir las emociones generadas en los jóvenes hacia emprendimientos de bebidas de Guayusa mediante aplicaciones de Neuromarketing para validar las respuestas del consumidor con su decisión de compra. Los participantes con edades entre 18 a 45 años, consumidores habituales de energizantes, degustaron ocho marcas de bebidas sin conocerlas, luego fueron expuestos a sus empaques. Mediante los datos de Diferencia Asimétrica Prefrontal obtenidos desde un electroencefalograma, fue posible corroborar la respuesta de bebida de mayor aceptación. La validación de aceptación a un nuevo emprendimiento desde la fisiología del cuerpo humano, implica un proceso moderno en la gestión para la comercialización de un nuevo producto, pues considera características específicas que determinan el comportamiento del consumidor. Los resultados prueban que las emociones juegan un rol importante, evidenciando que aún con ausencia en la percepción de orgullo en el tipo de bebida energizante tradicional o natural fue posible validar la marca con mayor aceptación entre los participantes.

Palabras Clave: Neuromarketing, emprendimientos, emociones, Guayusa, electroencefalograma 


\title{
Measuring Emotions Towards Guayusa Drink's Brands: Management Implications for Product Commercialization
}

\section{Dayana Jamile Romero-Buele}

Ingeniera en Administración de Empresas de la Universidad Técnica de Machala (UTMACH), Integrante del grupo de investigación OroResearch de la UTMACH, Dirección: Machala, El Oro, Ecuador

\section{Diana Maribel Domínguez-Maldonado}

Ingeniera en Administración de Empresas de la Universidad Técnica de Machala (UTMACH), Integrante del grupo de investigación OroResearch de la UTMACH, Dirección: Machala, El Oro, Ecuador

\section{Raquel Miroslava Tinoco-Egas}

Doctora en Análisis Económico y Estrategia Empresarial por la Universidade da Coruña, España, Master of Science in International Business

Development, Université de Neuachâtel, Suiza, Ingeniera en Gestión Empresarial Internacional, Profesora Titular de la Facultad de Ciencias

Empresariales de la Universidad Técnica de Machala (UTMACH), Coordinadora del grupo de investigación OroResearch de la UTMACH,

Dirección: Machala, El Oro, Ecuador

\section{Jonathan Neptali Herrera-Peña}

Doctor en Análisis Económico y Estrategia Empresarial por la Universidade

da Coruña, España, Magíster en Administración de Empresas por la

Universidad Técnica de Machala (UTMACH), Diplomado Superior en Docencia Universitaria por la Universidad Técnica de Machala (UTMACH), Docente Titular de la Facultad de Ciencias Empresariales de la Universidad

Técnica de Machala (UTMACH), Integrante del grupo de investigación

OroResearch de la UTMACH, Dirección: Machala, El Oro, Ecuador

\begin{abstract}
Emotion metrics from Neuromarketing devices validate consumer behavioral responses from the brain when exposed to sensorial stimuli. This study includes the analyze of consummer reactions when looking at the packaging and tasting the flavor of a natural energy drink Guayusa, used with an electroencephalography headset applied for getting marketing results as an analytical method from neuroscience to validate new products in the market before launching them. This descriptive-experimental research aims to measure the emotions generated in young people towards different brands of Guayusa natural drink through a Neuromarketing application to validate consumer responses regarding their final purchase decision. Participants between 18 to 45 years old with energy drinks consuming habits were considered for tasting eight new beverage brands and then being exposed to
\end{abstract}


their packaging to get acknowledgement of their presentation appealing. Using the prefrontal asymmetric difference data obtained from an electroencephalogram, it was possible to corroborate the most popular beverage response. Validating acceptance of a new brand name, or a new taste was possible from the physiology of the human body of participants; this process implies a modern step in the management and the commercialization of new products. Specific characteristics that determine the consumer's behavior were considered as part of new implications for the commercialization validation process. The results prove that emotions play an important role, evidencing that even with the absence of pride in the traditional or natural type of energy drink, it was possible to validate the brand with greater acceptance among the participants.

Keywords: Neuromarketing, entrerprises, emotions, Guayusa, electroencephalogram

\section{Introduction}

La Pacha Mama, concepto procedente de la lengua quechua que traducida al español, significa "Madre Tierra" provee al ser vivo las principales provisiones de subsistencia, siendo sus nativos los responsables de transmitir entre generaciones el conocimiento ancestral de cultivos y forma de preparación de productos autóctonos en cada región, que con el paso del tiempo se han convertido en una delicia para muchas personas. Sin embargo, las tradiciones locales se van perdiendo paulatinamente con la introducción de técnicas de marketing en productos externos que persuaden al consumo de otro tipo de bebidas o provisiones de subsistencia en los jóvenes. Hoy en día, se ha notado una disminución de la pertenencia o conocimiento cultural de los jóvenes hacia las bebidas de sus ancestros, razón por la cual se ha visto la necesidad de por medio de técnicas de neurociencia aplicadas al marketing, determinar el nivel de emociones que generan las bebidas ancestrales a consumidores de bebidas energéticas cuando degustan por primera vez productos de origen ancestral, sin haber sido expuesto a la marca de la bebida previamente, con el objetivo de identificar las reacciones del comportamiento del consumidor hacia la preferencia o el rechazo de la bebida ancestral. La electroencefalografía, es una de las técnicas utilizadas en neuromarketing, la teoría de la asimetría frontal quien fue desarrollada por Davidson y Rickman (1999) analiza los dos hemisferios cerebrales, la diferencia permitiría anticipar la conducta de aceptación o rechazo en el comportamiento del consumidor. Es importante mencionar que, el Neuromarketing es una herramienta novedosa, y explotada principalmente en marcas con grandes presupuestos de marketing, por lo tanto en las nuevas marcas de emprendimientos quienes hacen uso de 
las formas tradicionales de comercialización no tienen acceso por el costo que representaría utilizarlas.

Esta investigación exploratoria-experimental tiene como objetivo anticipar la respuesta de aceptación o rechazo por la diferencia asimétrica prefrontal dado por las emociones generadas en los jóvenes hacia emprendimientos de bebidas de Guayusa ante la exposición del sabor y la presentación de la marca por primera vez para validar la respuesta del consumidor a través de su decisión de compra. Adicionalmente se pretende determinar si los consumidores de bebidas energéticas tradicionales sienten orgullo al consumir una bebida energética elaborada a base una planta ancestral.

\section{Texto Principal:}

Según Vega (2016), el término Neuromarketing se lo empezó a usar por primera vez en el año 2002 por el ganador del Premio Nobel en economía Ale Smitds de la Universidad Erasmus de Rotterdam como una mejora a las estrategias de marketing tradicional empleando técnicas de neuroimagen en investigación de mercado y como un aporte a la neuroeconomía.

La digitalización constante y el aumento acelerado de la tecnología va dejando de lado la parte más humana, a día de hoy el neuromarketing demuestra que el marketing digital se vale principalmente de técnicas que evocan a las emociones para obtener mejores resultados de venta, por ello Cherubino, et al., (2019) describen específicamente como el estudio definido a "la aplicación de métodos neurocientíficos analiza y comprende el comportamiento humano en relación con los mercados y los intercambios comerciales" (pág. 2). Por su parte, Manzano, et al., (2012) mencionan que

Esta ciencia estudia todos los procesos mentales del consumidor relacionados con la percepción, memoria, aprendizaje, emoción y razón. A partir del entendimiento de estos elementos trata de explicar los factores que influyen y afectan a sus pensamientos, sentimientos, motivaciones, necesidades y deseos, para entender en última instancia qué define su comportamiento de compra. (pág. 72)

Lee, et al., (2007) definen al Neuromarketing como el campo de aplicación de la imagen neuronal a la investigación de mercados. Por su parte, Botello \& Suárez (2018) enfatizan que es considerada como una técnica con gran potencial, que combina ciencia y tecnología para generar soluciones a los problemas de marketing que se presentan actualmente, convirtiéndose así en una herramienta importante para diversas industrias dado que permite anticipar cuál será el nivel de aceptación que tendrá por ejemplo un producto nuevo en el mercado, mediante la proyección de resultados a través de datos obtenidos desde la reacción del cuerpo humano en un grupo de personas. 
Gracias al Neuromarketing ha sido posible demostrar que la decisión de compra no solo es un proceso racional (Naranjo, 2015), pues es importante comprender ciertos factores internos, especialmente las emociones, definidas como "el efecto producido por cambios fisiológicos significativos y su consiguiente interpretación subjetiva" (Coca, 2010, pág. 14). Mendoza, et al., (2018) establecen que, con la aplicabilidad de Neuromarketing se puede "determinar las reacciones de los clientes ante específicos estímulos comerciales porque se identifican las zonas cerebrales que se activan cuando ocurre el contacto con un determinado producto o atributo de una oferta" (pág. 72).

La actividad coordinada de miles de neuronas produce diferencias de potencial en el cuero cabelludo que pueden ser registradas utilizando electrodos en conjunción con amplificadores de señal. Es decir, colocando una serie de electrodos repartidos por la cabeza podemos hacernos una idea de en qué zonas de nuestro cerebro se está produciendo mayor actividad. (Monge-Benito \& Fernández-Guerra, 2011, pág. 23)

\section{Neuromarketing y la decisión de compra}

El proceso de decisión de compra es importante tanto para el cliente como para el comerciante, dado a que en él están inmersos diversos factores que son determinantes para la satisfacción de necesidades y posicionamiento de una empresa en el mercado. Alvarado-Marzano (2013) menciona que:

Las decisiones de compra son el resultado de emociones, si estas son positivas, el cliente compra; de lo contrario no lo hace. Diferentes investigaciones han demostrado que la satisfacción por un producto o servicio no garantiza la fidelidad del cliente. Se hace necesario algo más. Los clientes deben sentirse valorados y bien cuidados. Aquí es donde entra en juego el aporte del Neuromarketing, la importancia de las emociones de los consumidores. (pág. 6)

Braidot (2009) menciona que, el Neuromarketing es de tipo sensorial, recepta información a través de los sentidos por medio de logos, fotografías e imágenes, sonidos que atraen la atención del consumidor, facilitando su decisión de compra. Por su parte, Muñoz (2015) plantea que las emociones y sentimientos juegan un papel motivador y activador de experiencias en lo que a decisión de compra respecta. Para comprender el comportamiento del consumidor es imprescindible conocer su forma de pensar, sus gustos, qué cosas le llama la atención, sus colores preferidos entre otras cosas, lo cual no es fácil de analizar y por ello se tiende a tomar decisiones erróneas en la aplicación de estrategias de marketing (Tejada, et al., 2015). Por ello, se debe profundizar en el estudio del comportamiento de los consumidores, para un 
correcto proceso, pues todo inicia desde el diseño del producto o servicio, llegando hasta su adecuada comercialización, escogiendo correctamente el canal y el precio, la publicidad y las promociones que harán del mismo un éxito.

Actualmente, con el avance de las neurociencias se ha podido conocer que la decisión de compra, no es un proceso planeado de anteamano ni se la realiza por los atributos y características que presenta un producto o servicio, en su mayoría está ligada a factores emocionales que hacen que el producto sea más atractivo y el consumidor se sienta más familiarizado con el mismo (Martín \& Lázaro, 2013). La mayor parte de los consumidores, toman decisiones de compra desde el pensamiento no consciente (Paternina, 2016) pasando por alto los procesos racionales a la hora de comprar. Según (Morin, 2011) y (Fuller, 2014) indican "que el cerebro es quien guía el comportamiento de consumo de los individuos (como si fuera un centro de control)" (pág. 39). Convirtiéndose en foco de atención para los neurocientificos. A la vez que depende de factores externos e internos que son determinantes a la hora de adquirir un producto, siendo la motivación y percepción los factores internos primordiales.

- Percepción.- Según Baptista, et al., (2010) "la percepción es el proceso por el cual un individuo selecciona, organiza e interpreta estímulos para entender el mundo en forma coherente y con significado" (pág. 13). Es decir, a través del análisis del proceso perceptivo se puede obtener un mejor panorama de cómo los clientes reaccionan al entrar en contacto con ciertos estímulos de un producto o servicio, debido a que se está haciendo uso de todos los sentidos (vista, audición, olfato, gusto y tacto) lo que permite obtener respuestas de diferente índole y con ello identificar de mejor manera las necesidades de los clientes y encaminar estrategias generadoras de valor.

- Motivación.-La motivación se encuentra estrechamente vinculada con los deseos y necesidades de los consumidores. Durango, et al., (2016) indica que "La motivación es la función que actúa en el cerebro de un cliente y lo impulsa a actuar y esto está relacionado con una percepción de carencia o una satisfacción no satisfecha relacionada con un producto o servicio" (pág. 5).

Al determinar los diversos factores que intervienen en el comportamiento del consumidor y su decisión de compra, las empresas pueden detectar cuáles son los puntos claves que permitan implementar programas de mercadotecnia apegados a la realidad de los consumidores adaptando las propuestas comerciales a las especificaciones del segmento de mercado y con ello tener un crecimiento sostenido en cuanto a ventas y 
clientes. Actualmente, empresas como Coca-Cola, Levi-Strauss, Ford, Delta Airlines, DaimlerChrysler entre otras, consideran al Neuromarketing como su herramienta principal la investigación de mercados y estudios del consumidor. (Coca, 2010)

\section{Experimentos con EEG}

El electroencefalograma conocido por sus siglas en inlés EEG "es la representación gráfica y digital de las oscilaciones que muestra la actividad eléctrica del cerebro, al ser registrada mediante electrodos colocados encima del cuero cabelludo en distintas regiones de la cabeza" Carrillo \& et al. (2013). En muchas investigaciones se utiliza el electroencefalograma (EEG) para monitorizar los efectos de cambio existentes sobre la dinámica cerebral mediante un estudio específico, la sensibilidad del EEG en la identificación de alteraciones producidas por un determinado ejercicio, puede mejorarse empleando métodos de análisis cuantitativo (qEEG), por ello, los cambios en las variables del qEEG pueden utilizarse para investigar mecanismos de acción, así como para monitorizar y posiblemente predecir su eficacia. (Machado, et al., (2009) siendo el Emotiv Epoc uno de los instrumentos mayormente utilizado dentro de este campo investigativo.

Según Goudiaby \& et al. (2020) el Emotiv Epoc+, es una diadema que permite detectar las ondas cerebrales generadas por las acciones mentales. El dispositivo cuenta con sensores superficiales los cuales se ubican en el cuero cabelludo, posee 14 canales de lectura AF3, F7, F3, FC5, T7, P7, O1, O2, P8, T8, FC6, F4, F8, AF4, los cuales se ubican en la parte frontal, parietal, occipital y temporal de la cabeza, y 2 canales de referencia colocados detrás de las orejas. Las señales obtenidas por los sensores son transmitidas por bluetooth a un computador a través de una memoria externa USB. Es importante que los sensores pasen por un proceso previo de hidratación, saturando cada uno de ellos con una solución salina que permite una mejor transmisión. El Emotiv Epoc+ adquiere datos de la subdivisión más importante del encéfalo, es decir, la corteza cerebral, que contiene la gran mayoría de neuronas existentes en el cerebro humano. La corteza cerebral contiene muchas fisuras o pliegues, llamados surcos, que se utilizan para dividir la corteza en lóbulos. La distribución de los diferentes lóbulos comprende el lóbulo frontal, lóbulo parietal, lóbulo occipital y lóbulo temporal.

- El lóbulo frontal: controla los impulsos, la producción del lenguaje, la memoria funcional, las funciones motoras, el comportamiento sexual, el control de las emociones, la socialización y la espontaneidad. Da la capacidad de razonar y de resolver problemas. Asiste en la planificación, coordinación control y ejecución de la conducta. 
- El lóbulo parietal: es el encargado de las percepciones sensoriales procedentes de varias partes del cuerpo humano, como la sensibilidad, el tacto, la percepción, la presión, la temperatura y el dolor. También interviene en el conocimiento de los números y sus relaciones.

- El lóbulo occipital: es el encargado de la producción de imágenes. En él reside la corteza visual, por lo que está implicado en nuestra capacidad para ver e interpretar lo que vemos.

- El lóbulo temporal: es el encargado de desempeñar un papel primordial en tareas visuales complejas, como el reconocimiento de caras. Recibe y procesa información de los oídos. Está encargado de la audición, equilibrio y coordinación. Es el centro primario del olfato. También regula emociones y motivaciones, como la ansiedad, el placer y la ira. Está implicado en las funciones de la memoria, el recuerdo de palabras y el nombre de los objetos.

El software Emotiv Pro proporciona seis medidas básicas de desempeño mental estimadas directamente a partir de la actividad cerebral; estrés, compromiso, interés, excitación, relajación y enfoque (CorredorCamargo, Peña-Cortés, \& Pardo-García, 2019):

- Compromiso (Engagement): Mide la voluntad, la atención y concentración con la que el individuo realiza la actividad.

- Interés (Interest): Es el grado de aceptación, atracción o a la vez el rechazo hacia la actividad que ejecuta el usuario. Aquellos valores bajos muestran que el individuo siente disgusto por la actividad, por el contrario, si se presentan valores altos de interés nos muestran que el individuo se siente conectado y vinculado con la actividad; en ocasiones también se pueden visualizar valores intermedios los cuales podemos interpretar que no siente ni agrado ni disgusto por la actividad. El interés muestra una relación con el goce de la tarea realizada.

- Excitación (Excitement): Estado que se muestran en valores altos, bajos e intermedios de los cuales se interpreta el nivel de pasión o entusiasmo emocional, se puede puntualizar como aquel estado con mayor actividad físico-mental.

- Enfoque (Focus): Nivel de atención fija hacia la actividad. Evalúa el interés presentado durante la actividad, pudiéndose ver el mismo perjudicado por la distracción.

- Estrés (Stress): Nivel de comodidad con la actividad. El alto grado de estrés puede indicar que el inconformismo o la inhabilidad para la realización de tareas complejas, causando, por lo tanto, agobio y aturdimiento a resultados negativos por no cumplir adecuadamente con 
la actividad. Por el contrario, al mostrarse un nivel bajo o intermedio de estrés mejora positivamente la estabilidad emocional y la efectividad de agrado a la actividad.

- Relajación (Relaxation): Es el alcance de tranquilidad alcanzado durante la actividad. Se puede mencionar como el estado de descanso y restauración de la concentración.

De particular interés para la investigación de Neuromarketing son los ritmos alfa y beta. El primero se relaciona directamente con las emociones (asimetría pre frontal mientras que el ritmo beta se relaciona con el estado de atención activa, estado de alerta y expectativa de recompensa. Los investigadores miden el nivel de disminución del ritmo alfa para ser sustituido por el ritmo beta, una situación característica de la actividad cognitiva. (Vega, 2016).

Uno de los estudios más relevantes en este ámbito, es la comparación realizada a las bebidas gaseosas Coca-Cola y Pepsi, pues se demostró que, las personas pese a que preferían el sabor de Pepsi, a la hora de consumir un producto se inclinaban por Coca-Cola, con lo cual se constató que la marca tiene mayor influencia en la mente del consumidor. (Lee, et al., 2007).

Grandes empresas como Google y Hyundai utilizaron la técnica de electroencefalografía (EEG). La primera para hacer una comparativa de los diversos sitios web y determinar el contenido más atractivo y relevante para los usuarios, mientras que Hyundai recurrió a esta técnica con la finalidad de comprender los gustos y preferencias que tienen los consumidores respecto a los automóviles y sus motivaciones para comprar y en base a los hallazgos realizar ajustes de diseño. (ESCO Universitas, 2020).

Para Ruiz y Arranz citado Coca (2010) "The Role of Emotions in Marketing" es uno de los escritos más importantes referentes al papel que juegan las emociones en las estrategias de marketing, considera que las emociones tienen un rol importante en la vida de las personas y por consiguiente el comportamiento del consumidor. Un claro ejemplo son los diversos congresos que se desarrollan considerando a las emociones como un factor principal para el desarrollo de estrategias de marketing. Para medir las emociones, se debe fundamentar en una base teórica. Según Álvarez, et al., (2000) en la medición de las emociones se debe considerar varios componentes entre los cuales se destacan (fisiológico, comportamental y cognitivo) cada uno de ellos por separado. Además, es importante considerar otros aspectos relacionados netamente con la personalidad. 


\section{Emprendimientos que rescatan el conocimiento ancestral}

Vislumbrar las razones de motivación para la creación de nuevos emprendimientos admite comprender que el hombre se caracteriza por su tenacidad en asumir riesgos con el objetivo de generar oportunidades de crecimiento económico y así comprobar la voluntad de cada persona a incursionar con un emprendimiento. Existen múltiples factores los cuales inciden en ésta decisión, los motivos e intenciones según los rasgos de personalidad juegan un papel importante en la creación de una empresa y el lanzamiento de nuevos productos. (Zhao \& Seibert, 2006).

Es fundamental analizar puntualmente el estado actual de los productos o servicios existentes en el medio, pensando siempre más allá a la hora de generar una necesidad para futuros clientes. Si bien es cierto, el mundo actual está copado de productos y servicios categóricos, algunos de ellos perjudiciales para la salud y el ambiente, sin embargo, muchos visionarios han visto la necesidad de crear productos existentes apegados a la naturaleza, es decir, ofertar productos cada vez más sanos y libre de químicos como nueva alternativa de consumo (Pilcon, 2013). Es aquí donde nacen las diferentes bebidas energéticas a base de una planta natural como lo es la Guayusa (Ilex Guayusa) ayudándonos a "tener energía y estar alegre durante todo el día. Tradicionalmente se usa para desinflamar la próstata, purgante del sistema digestivo, las mujeres toman su infusión como remedio contra la infertilidad, contra la diarrea y curación de heridas" (Shaman Amazónico, 2016).

Ilex Guayusa Loes, pertenece a la familia Aquifoliaceae; es conocida comúnmente como Guayusa, planta sagrada de las nacionalidades indígenas, resalta su identidad y patrimonio cultural; se la puede localizar principalmente en regiones tropicales y subtropicales de América y Oceanía. (Brücher, 2012). En el Ecuador, a la planta de Guayusa se la puede encontrar en las provincias que conforman la región amazónica, así también, en las provincias de Pichincha y Tungurahua (Jorgensen \& León-Yánez, 1999). Pérez \& et al. (2017) manifiestan que, a la hoja de Guayusa se la puede consumir como una infusión y como un estimulante leve. Habitualmente, es empleada para tratar problemas de infertilidad, combatiendo desequilibrios hormonales presentados en la mujer; ayuda a resolver problemas de escalofrío e infecciones venéreas (García, 1975). Por el alto contenido de cafeína, teobromina y otros estimulantes, también es utilizada en la elaboración de bebidas energéticas naturales, dado que, no contiene taurina ni ninguna otra sustancia química dañina para la salud (Sidali, Yepez, \& Garrido-Pérez, 2016). Ecuador país mega diverso y pluricultural, ha tenido grandes herencias en lo que respecta a productos naturales, por eso, al día de hoy, se ha visto un incremento en emprendimientos de origen ancestral, con los cuales se pretende conservar las tradiciones. Sin embargo, en las nuevas generaciones existe un limitado conocimiento en cuanto a las herencias generadas por nuestros 
ancestros, disminuyendo nuestra gran diversidad cultural (Leiva \& Díaz, 2013). Hoy en día, se considera a la globalización como una fuerte amenaza para la preservación de valores culturales ancestrales, perdiendo la esencia de vincular a la colectividad a compartir costumbres y tradiciones; planteando como posible solución la inclusión al conocimiento ancestral en la convivencia social. De igual manera, dado a que actualmente, los niños y jóvenes emplean gran parte de su tiempo en actividades educativas, han perdido la posibilidad de adquirir conocimientos ancestrales a través de la observación, experimentación y transmisión intrafamiliar; razón por la cual, se cree pertinente que los programas educativos y extracurriculares realicen actividades para resguardar dichas tradiciones, a través del conocimiento y valoración de la cultura e historia y con ello infundir orgullo a lo ancestral en las nuevas generaciones (Food and Agriculture Organization of the United Nations, 2017).

¿Orgullo es una emoción o sentimiento?

Existe una estrecha relación entre emociones y sentimientos. Según Damasio (2002):

Las emociones se manifiestan a partir de un estímulo externo. La información sensorial se transmite por medio de las vías que van del cuerpo a la médula espinal. A este nivel se generan sinapsis que informan al cerebro por medio de diferentes circuitos neuronales y la respuesta a dicho estímulo se produce mandando información hormonal (por glándulas endócrinas) o muscular (latidos del corazón). El sentimiento es una idea que se tiene en el cerebro, es un estado mental diferente de las emociones, que se manifiestan en reacciones corporales ante estímulos capaces de desencadenar. (pág. 13)

Weiner (1985) manifiesta que, la palabra orgullo es una emoción positiva que se siente después de alcanzar ciertos objetivos o metas. Por lo general, se emplea para demostrar a los demás los méritos alcanzados. Mientras que Carrasquel (2009) define al término orgullo como un sentimiento de confianza que resalta la importancia de la cultura y tradiciones heredadas de nuestros ancestros, y que permiten engrandecer a un pueblo, por ello, en la presente investigación se procede a analizar mediante datos obtenidos a través del electroencefalograma si los participantes en la misma sienten o no orgullo hacia un producto de origen ancestral.

Sin embargo, para Parrot citado en Reidl \& Jurado (2016) el orgullo, la pena y vergüenza, son consideradas emociones de auto-conciencia puesto que, se van desarrollando conjuntamente con la socialización, con el fin de evaluar la imagen que una persona tiene sobre sí misma, frente a lo que los demás creen o esperan de ella. Por lo que, muchas de las veces las personas tienden a ocultar su verdadera identidad. 


\section{Materiales y Métodos \\ Diseño Experimental}

En la medición e identificación de las emociones generadas en los jóvenes hacia emprendimientos de bebidas de Guayusa se procedió a realizar un estudio exploratorio-experimental.

\section{Participantes}

Se determinaron las emociones generadas de ocho marcas de bebidas de Guayusa (sabor natural, de limón y de frutos rojos) a diez voluntarios entre 18 a 45 años de edad, entre ellos seis hombres y cuatro mujeres, consumidores frecuentes de bebidas energizantes, permitiendo validar desde el cerebro la marca con mayor preferencia de los participantes.

\section{Instrumentos}

Después de haber obtenido la firma de aceptación y consentimiento voluntario a la participación y explicación de este protocolo de investigación (de acuerdo a la declaración de Helsinki), se procedió a tomar los datos personales y generales sobre el conocimiento de bebidas energéticas convencionales.

Para la recolección de dicha información se utilizó una encuesta desarrollada en la plataforma de Google Forms, mientras que la recolección de datos fisiológicos se realizó a partir de la actividad eléctrica generada por las neuronas en el interior del cerebro, obtenidas a través del cráneo por medio de un casco de electrodos (Casco Emotiv Epoc+ de 16 canales) conectados en la superficie del cuero cabelludo y de esta manera determinar a través de un software (Emotiv Pro) el desempeño mental mediante emociones. El principio estratégico en la investigación aplicando electroencefalograma. Los datos se mantuvieron en confidencialidad durante todo el proceso. Colocado el casco Emotiv en el cráneo del participante, degustaron uno por uno en un orden aleatorio ocho sabores de bebidas energéticas (emprendimientos de bebidas de Guayusa) de diferentes marcas. Los participantes valoraron el sabor y asignaron el orden de su preferencia (sin conocer previamente el envase) para cada una de las marcas, y así lo hicieron también para la presentación de los envases con sus etiquetas. Posteriormente, se pide tomar una decisión de compra y escoger una bebida energética entre todas las opciones y consecutivamente se pregunta también si siente orgullo de las bebidas energéticas de origen ancestral.

Finalmente, para el análisis estadístico fue necesario hacer uso del programa estadístico SPSS (Statistical Package for Social Sciences) en la versión 22. A través de la aplicación de Regresión Logística Binaria y R cuadrado de Nagelkerke, se pudo analizar la relación de la diferencia asimétrica prefrontal con la decisión de compra de los participantes evaluados. 


\section{Análisis y Resultados}

\section{Identificación de emociones}

A continuación, se pueden identificar las emociones generadas en los jóvenes hacia emprendimientos de bebidas de Guayusa mediante la aplicación del casco EMOTIV y su software Emotiv Pro que identifica las emociones para cada bebida.

\begin{tabular}{|l|l|l|l|l|}
\hline \multicolumn{5}{|c|}{ Tabla 1. Emociones generadas en los participantes } \\
\hline \multirow{3}{*}{ Emoción } & $\begin{array}{l}\text { Promedio } \\
\text { de } \\
\text { Intensidad }\end{array}$ & $\begin{array}{l}\text { Todos } \\
\text { estímulos }\end{array}$ & Presentación & Sabor \\
\hline \multirow{3}{*}{ Estrés } & Menor & Iwia Original & Iwia Jamaica & Iwia Original \\
\cline { 2 - 6 } & Mayor & Misha Limón & Misha Limón & Iwia Limón \\
\hline \multirow{2}{*}{ Interés } & Menor & Liv Frutos Rojos & Liv Frutos Rojos & $\begin{array}{l}\text { Liv } \\
\text { Rojos }\end{array}$ \\
\hline \multirow{2}{*}{ Excitación } & Mayor & Misha Limón & Iwia Original & $\begin{array}{l}\text { Misha Frutos } \\
\text { Rojos }\end{array}$ \\
\cline { 2 - 6 } & Menor & Misha Frutos Rojos & Misha Frutos Rojos & Iwia Original \\
\cline { 2 - 6 } & Mayor & Guari Frutos Rojos & Guari Frutos Rojos & Iwia Limón \\
\cline { 2 - 6 } Enfoque & Mayor & Guari Frutos Rojos & Guari Frutos Rojos & Misha Limón \\
\cline { 2 - 6 } & Menor & Iwia Original & Iwia Original & Iwia Original \\
\hline \multirow{2}{*}{ Relajación } & Menor & Guari Frutos Rojos & Misha Frutos Rojos & $\begin{array}{l}\text { Misha Frutos } \\
\text { Rojos }\end{array}$ \\
\cline { 2 - 6 } & Mayor & Misha Frutos Rojos & Iwia Jamaica & $\begin{array}{l}\text { Misha Frutos } \\
\text { Rojos }\end{array}$ \\
\hline & & & Iwia Original & Iwia Limón \\
\hline
\end{tabular}

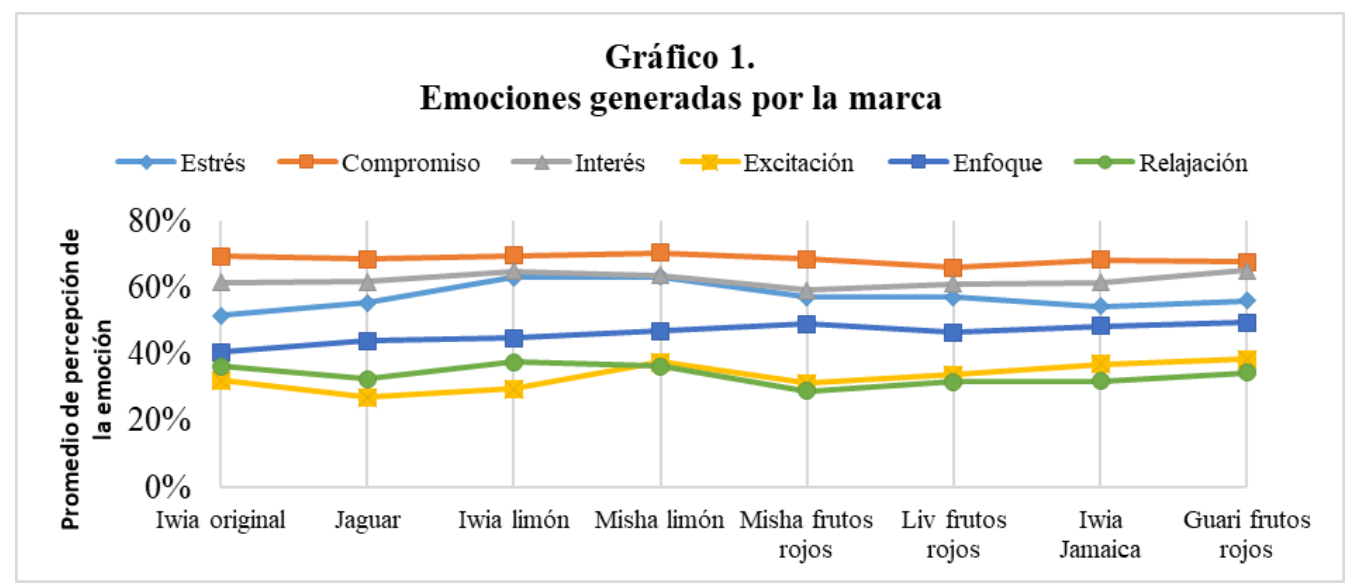




\section{Valoración de la Marca}

Mediante un cuestionario se solicitó que valore la marca inmediatamente después de haber recibido el estímulo, de los que se obtuvieron los siguientes resultados:

\begin{tabular}{|l|l|l|l|l|}
\hline \multicolumn{4}{|l|}{ Tabla 2. Valoración de la marca } \\
\hline $\begin{array}{l}\text { Acción } \\
\text { consciente }\end{array}$ & $\begin{array}{l}\text { Promedio de } \\
\text { Intensidad }\end{array}$ & Todos los estímulos & Presentación & Sabor \\
\hline $\begin{array}{l}\text { Valoración de } \\
\text { Marca }\end{array}$ & Menor & Iwia Limón & Iwia Limón & Liv Frutos Rojos \\
\cline { 2 - 5 } & Mayor & Jaguar & Liv Frutos Rojos & Guari Frutos Rojos \\
\hline
\end{tabular}

(Fuente: elaboración propia.)

\section{Hipótesis}

A continuación, se procede a identificar las siguientes hipótesis:

- H1. El orgullo depende de las emociones generadas por los estímulos. Según la información obtenida con un $\mathrm{R}$ cuadrado de Nagelkerke de $84.3 \%$, que identifica el porcentaje de predicción de los resultados, se obtuvo mediante una regresión logística binaria, que el sentir o no orgullo a la bebida de origen ancestral depende de las emociones generadas durante el protocolo en el que fueron expuestos los estímulos de presentación y sabor de todas las marcas, y son específicamente las emociones de excitación $(\beta=0.004, p=0.029)$ y estrés $(\beta=-0.006, p=0.031)$ de las que el orgullo dependen. Estos resultados fueron obtenidos manteniendo al nivel de orgullo por la ingesta de bebidas convencionales energéticas como variable de control.

- H2. La diferencia asimétrica prefrontal positiva se relaciona con la decisión de compra de la mayoría de los participantes. Como podemos observar en la tabla $\mathrm{N}^{\circ} 3$ la marca de preferencia de los participantes es JAGUAR, y en ellos se visualiza una diferencia asimétrica positiva, lo que relaciona la diferencia positiva con la decisión de compra de la mayoría de los participantes. A excepción del participante $\mathrm{N}^{\circ} 3$ siendo el único que desde el inicio de la investigación dio una respuesta negativa respecto a sentir orgullo hacia las bebidas ancestrales energéticas con sabor a Guayusa, presenta una diferencia negativa prefrontal asimétrica, posteriormente analizando los resultados obtenidos a través del casco observamos que su respuesta sigue siendo negativa, manteniendo en pie su posición de no sentir orgullo hacia éste tipo de bebidas, pero quien manifestó sin embargo adquirir el producto por su sabor y presentación a pesar de no tener orgullo hacia las bebidas de origen ancestral. 


\section{Diferencia Asimétrica}

La diferencia asimétrica prefrontal es una de las aplicaciones más utilizadas en los estudios de Electroencefalograma, mide las regiones frontales derecha e izquierda del cerebro (AF3_AF4). AF3 corresponde al lóbulo frontal izquierdo en tanto que AF4 corresponde al lóbulo frontal derecho (Ausin, 2020). Los electrodos AF3 y AF4, se relacionan con las secuencias de comportamientos y toma de decisiones e incluyen movimientos oculares, razonamiento, valoraciones emocionales (Jodar-Vicente, 2014). Por medio de esta técnica se puede comprender cuando la gente se interesa o rechaza un producto, pudiendo así predecir el éxito del mismo.

\begin{tabular}{|c|c|c|c|}
\hline \multicolumn{4}{|c|}{ Tabla 3. Diferencia Asimétrica } \\
\hline Participante & $\begin{array}{l}\text { Diferencia asimétrica } \\
\text { AF3_AF4 por la marca }\end{array}$ & $\begin{array}{l}\text { Decisión de compra del } \\
\text { participante }\end{array}$ & $\begin{array}{l}\text { Siente orgullo hacia } \\
\text { las bebidas energéticas } \\
\text { con origen ancestral }\end{array}$ \\
\hline 1 & $8 * 10^{4}(0.000763154)$ & Jaguar & $\mathrm{Si}$ \\
\hline 2 & $\begin{array}{c}2 * 10^{-3} \\
-0.002300635\end{array}$ & Liv Guayusa & $\mathrm{Si}$ \\
\hline 3 & $\begin{array}{r}6 * 10^{-5} \\
-0.0000581555\end{array}$ & Jaguar & No \\
\hline 4 & $\begin{array}{r}2 * 10^{-5} \\
-0.0000156695\end{array}$ & Guari frutos rojos & $\mathrm{Si}$ \\
\hline 5 & $\begin{array}{r}2 * 10^{-3} \\
-0.002474396\end{array}$ & Misha frutos rojos & $\mathrm{Si}$ \\
\hline 6 & $\begin{array}{r}4 * 10^{3} \\
0.003863377\end{array}$ & Jaguar & $\mathrm{Si}$ \\
\hline 7 & $\begin{array}{r}4 * 10^{2} \\
0.035920731\end{array}$ & Iwia frutos rojos jamaica & $\mathrm{Si}$ \\
\hline 8 & $\begin{array}{r}1 * 10^{-2} \\
-0.013428739\end{array}$ & Iwia original & $\mathrm{Si}$ \\
\hline 9 & $\begin{array}{r}2 * 10^{3} \\
0.001846884\end{array}$ & Jaguar & $\mathrm{Si}$ \\
\hline 10 & $\begin{array}{r}6 * 10^{4} \\
0.000617128\end{array}$ & Jaguar & $\mathrm{Si}$ \\
\hline
\end{tabular}

(Fuente: elaboración propia.)

\section{Discusiones y Conclusiones}

El neuromarketing tiene gran relevancia en el ámbito empresarial, tanto en empresas ya establecidas como en los emprendimientos, a través de los diferentes métodos de análisis, los cuales contribuyen positivamente a la disminución del rango de error a lo que se refiere a investigación de mercados y estudios del consumidor. El utilizar nuevas herramientas de investigación han contribuido a una mejor toma de decisiones al momento de crear nuevas estrategias, tomando en cuenta el área sociodemográfica (grado de escolaridad, ingresos, lugar de residencia, composición familiar, estrato socioeconómico, estado civil, raza, ocupación, área de trabajo, edad, 
sexo) donde se desarrolle la investigación, reduciendo en gran parte el fracaso a la no aceptación de nuevos productos.

Como demuestra la investigación, con el $84.3 \%$ obtenida con un $\mathrm{R}$ cuadrado de Nagelkerke se logró determinar que el estrés y la excitación son las emociones de la cual el orgullo depende, en relación a las bebidas energéticas ancestrales a base de Guayusa.

Podemos ver que, tomando para investigación el AF3 Y AF4 se estableció que la diferencia asimétrica prefrontal positiva se relaciona con la decisión de compra de la mitad de los jóvenes participantes evaluados, los cuales presentaron preferencia por la marca Jaguar, comprobando por lo tanto, el comportamiento favorable o desfavorable hacia el estímulo.

Finalmente, a través de autoevaluaciones (cuestionarios físicos) se puedo determinar que gran parte de los participantes se sienten orgullos de consumir una bebida energética elaborada a base de un producto ancestral a excepción del participante $\mathrm{N}^{\circ} 3$ quien contestó desfavorablemente a sentir orgullo hacia las bebidas ancestrales energéticas con sabor a Guayusa; con lo cual aprueban esta nueva idea de negocio.

\section{References:}

1. Monge-Benito, S., \& Fernández-Guerra, V. (2011). Neuromarketing: Tecnologías, Mercado y Retos. Pensar la Publicidad(2), 19-42. doi:http://dx.doi.org/10.5209/rev_PEPU.2011.v5.n2.37862

2. Damasio, A. (2002). Le sentiment même de soi, corps, émotions, conscience. París: Éditions Odile Jacob,. Obtenido de https://bit.ly/3lxbppR

3. Reidl, L., \& Jurado, S. (2016). Culpa y vergüenza. México D.F: Universidad Nacional Autónoma de México UNAM. Obtenido de https://bit.ly/2QCtdC4

4. Brücher, H. (2012). Useful Plants of Neotropical Origen and their Wild Relatives. Berlin, Alemania: Springer Science \& Business Media. Obtenido de https://bit.ly/2YMiCZu

5. Jorgensen, P., \& León-Yánez, S. (1999). Monographs in Systematic Botany from The Missouri Botanical Garden. Catalogue of the Vascular Plants of Ecuador., 75, 1-1181. Obtenido de https://bit.ly/2ECruKs

6. Food and Agriculture Organization of the United Nations. (2017). Juventud rural y empleo decente en América Latina. Santiago: Food \& Agriculture Org. Obtenido de https://bit.ly/2YHO5vO

7. Davidson, R., \& Rickman, M. (1999). Behavioral inhibition and the emotional circuitry of the brain: Stability and plasticity during the early childhood years. New York: In Schmidt \& J. Shulkin (Eds.). doi:https://doi.org/10.1093/acprof:oso/9780195118872.003.0005 
8. Lee, N., Broderick , A., \& Chamberlain, L. (2007). What is 'neuromarketing'? A discussion and agenda for future research. International Journal of Psychophysiology, 63(2), 199-204. Obtenido de https://bit.ly/3lrJwzJ

9. Botello, S., \& Suárez , K. (2018). Tendencias de Investigación en Neuromarketing. Cuadernos Latinoamericanos de Administración, XIV(27). Obtenido de https://bit.ly/2G9bhwP

10. Weiner, B. (1985). An attributional theory of achievement motiva-tion and emotion. Psychological Review, 92(4), 548-573. Obtenido de https://bit.ly/3lxbppR

11. Carrasquel, D. (2009). Visión de la lengua yarura (Vol. 73). Caracas: Santillana.

12. García, H. (1975). Flora medicinal de Botánica Médica. Bogotá: Editorial Imprenta Nacional Bogotá. Obtenido de https://bit.ly/3jrXb85

13. Zhao, E., \& Seibert, S. (2006). The Big Five personality dimensions and entrepreneurial status: A meta-analytical review. Journal of Applied Psychology(2), 259-271. Obtenido de https://bit.ly/2Eu2eGn

14. Leiva, O., \& Díaz, R. (2013). Tras las huellas alimentarias de nuestros ancestros. "Sembrando el pasado y cultivando el presente en armonía con la Madre Tierra". Revista Electrónica Educare, 17(2), 81-89. Obtenido de https://bit.ly/3ln $5 \mathrm{vHU}$

15. Corredor-Camargo, J., Peña-Cortés, C., \& Pardo-García, A. (2019). Evaluación de las emociones de usuarios en tareas con realimentación háptica utilizado el dispositivo Emotiv Insight. INGE CUC(1), 9-16. doi:http://dx.doi.org/10.17981/ingecuc.15.1.2019.01

16. ESCO Universitas. (2020). Escuela Internacional de Neurociencia y Empresa. Obtenido de Neuromarketing, aprende de estos ejemplos: https://www.escoeuniversitas.com/neuromarketing-ejemplos/

17. Alvarado-Marsano, L. (2013). Neuromarketing, ciencia al servicio del conocimiento. Sinergia e Innovación, 1(2).

18. Muñoz, M. (2015). Conceptualización del neuromarketing: su relación con el mix de marketing y el comportamiento del consumidor. $R A N$ Revista Academia \& Negocios, 1(2), 103-118. Obtenido de https://papers.ssrn.com/sol3/papers.cfm?abstract_id=2777534

19. Paternina, M. (2016). Neuromarketing como herramienta de posicionamiento de marcas. In Vestigium Ire, 10(2), 166 - 180.

20. Salas, H. (2018). Neuromarketing: Explorando la mente del consumidor. Revista Científica de la UCSA, 5(2), 36-44.

21. Sidali, K., Yepez, P., \& Garrido-Pérez, E. (2016). Food Tourism in Indigenous Settings as a Strategy of Sustainable Development: The 
Case of Ilex guayusa Loes. in the Ecuadorian Amazon. Sustainability(967), 1-17. Obtenido de https://bit.ly/34KFQmS

22. Naranjo, P. (2015). Introducción al Neuromarketing. Revista de Investigación en Ciencias Sociales y Humanidades(2), 1-14. Obtenido de https://bit.ly/3gE58Fp

23. Manzano, R., Gavilán, D., Avello, M., Abril, C., \& Serra, T. (2012). Marketing sensorial. Comunicar con los Sentidos en el Punto de Venta. Madrid: Pearson. Obtenido de https://bit.ly/34HQ9b7

24. Vega, O. (2016). Neuromarketing y la ética en la investigación de mercados. Ingeniería Industrial. Actualidad y Nuevas Tendencias, $V(16), 69-82$. Obtenido de https://bit.ly/3jecEbM

25. Coca, A. (2010). Neuromarketing: Las Emociones y el Comportamiento de Compra. Perspectivas(25), 9-24. Obtenido de https://bit.ly/3jm3VUU

26. Mendoza, E., Boza, J., Escobar, H., \& Macías, G. (2018). El Neuromarketing y las emociones, factor de éxito en la construcción de marcas en los emprendimientos. Cumbres(1), 69-81. Obtenido de https://bit.ly/2QvWWwe

27. Martín, P., \& Lázaro, C. (2013). Neuromarketing: la última frontera de comercialización. Gestión 57, 14-18.

28. Baptista, M., León, M., \& Mora, C. (2010). Neuromarketing: conocer al cliente por sus percepciones. Tec Empresarial(3), 9-19.

29. Durango, J., Espinal, N., \& Giraldo, M. (2016). El neuromarketing y la conducta del comprador. Revista electrónica Psyconex, 8(2), 1-8.

30. Coca, A. (2010). Neuromarketing: Las Emociones y el Comportamiento de Compra. Perspectivas(25), 9-24. Obtenido de https://bit.ly/3jm3VUU

31. Cherubino, P., Martinez-Levy, A., Caratù, M., Cartocci, G., Di Flumer, G., Modica, E., \& Trettel, A. (2019). Consumer Behaviour through the Eyes of Neurophysiological Measures: State-of-the-Art and Future Trends. Computational Intelligence and Neurocience, 1-41. doi:10.1155/2019/1976847

32. Álvarez, M., Bisquerra, R., Fita, E., \& Martínez, F. (2000). Evaluación de programas de educación emocional. Revista de Investigación Educativa(2), 587-599. Obtenido de https://bit.ly/2EBufM3

33. Pérez, J. M., Cardona, W., Urango, L., Alzate, F., Rojano, B., \& Maldonado, M. (2017). Aspectos nutricionales y fisioquímicos de Ilex laurina Kunth (Aquifoliaceae): un estudio comparativo con Ilex paraguariensis. Perspectivas en Nutrición Humana(1), 41-54. Obtenido de https://bit.ly/2ELSeYM 
34. Ausin, J. (2020). Técnicas del Neuromarketing. Obtenido de Por qué y cómo el neuromarketing mide la actividad cerebral: https://josemanuelausin.com/tecnicas-del-neuromarketing/eeg/

35. Machado, D., Bastos, V., Cunha, M., Velasques, B., Machado, S., Basile, L., \& Ribeiro, P. (2009). Efectos del bromacepam en el desarrollo de una actividad sensoriomotora: un estudio electroencefalográfico. Revista de Neurología(6), 295-299. doi:https://doi.org/10.33588/rn.4906.2008471

36. Pilcon, W. (2013). Mercados Cautivos para Productos Ancestrales. Revista Ciencia UNEMI(10), 82 - 93.

37. Jodar-Vicente, M. (2014). Funciones cognitivas del lóbulo frontal. Revista de neurología, 39(2), 178 - 182.

38. Braidot, N. (2009). Neuromarketing ¿Por qué tus clientes se acuestan con otros si dicen que les gustas tu? (Vol. 8). Gestión 2000.

39. Morin, C. (2011). Neuromarketing: la nueva ciencia del comportamiento del consumidor. SpringerLink .

40. Fuller, S. (2014). Neuroscience, Neurohistory, and the History of Science: A Tale of Two Brain Images. ISIS, 105(1), 100-109.

41. Carrillo, P., Ramirez, P., \& Magaña, K. (2013). Neurobiología del sueño y su importancia oara el estudiante universitario. Revista de la Facultad de Medicina de la UNAM(4), 5-15. Obtenido de https://bit.ly/31BDGUk

42. Goudiaby, B., Othmani, A., \& Naït-ali, A. (2020). EEG Biometrics for Person Verification. Research Gate, 45-69. Obtenido de https://bit.ly/3jTSq7B

43. Shaman Amazónico. (2016). Aplicaciones médicas tradicionales de la guayusa en la amazonía ecuatoriana. Ecuador: Villacís, J. (Ed.). 